For submission to J Proteome Res

\title{
Combined peptidomic and proteomic analysis of electrically stimulated and manually dissected venom from the South American bullet ant Paraponera clavata
}

Samira R. Ailia, Axel Touchard ${ }^{b}$, Frédéric Petitclerc ${ }^{b}$, Alain Dejean ${ }^{b, c}$, Jérôme Orivel ${ }^{b}$, Matthew P. Padulad, Pierre Escoubas ${ }^{e}$, Graham M. Nicholson ${ }^{a^{*}}$

${ }^{a}$ Neurotoxin Research Group, School of Life Sciences, University of Technology Sydney, NSW 2007, Australia

${ }^{b}$ CNRS, UMR Ecologie des Forêts de Guyane (EcoFoG), AgroParisTech, Cirad, INRA, Université des Antilles, Université de Guyane, Université des Antilles, 97310 Kourou, France

${ }^{\mathrm{c}}$ Ecolab, Université de Toulouse, CNRS, INPT, UPS, Toulouse, France

dProteomics Core Facility, Faculty of Science, University of Technology Sydney, NSW 2007,

Australia

'VenomeTech, 473 Route des Dolines - Villa 3, Valbonne 06560, France

*Author for correspondence: Graham Nicholson, Neurotoxin Research Group, School of Life Sciences, University of Technology Sydney, NSW 2007, Australia.

Tel: (+61 2) 9514 2230; Fax: (+61 2) 95141656

E-mail: Graham.Nicholson@uts.edu.au (G. Nicholson)

Keywords: Proteome, Peptidome, Ants, Bullet Ant, Venom, MALDI-TOF MS, nanoLC-ESIQTOF MS/MS, 2D-PAGE, Paraponera clavata, dissection, electrical stimulation 


\begin{abstract}
Ants have evolved venoms rich in peptides and proteins used for predation, defence and communication. However, they remain extremely understudied due to the minimal amount of venom secreted by each ant. The present study investigated the differences in the proteome and peptidome of the venom from the bullet ant, Paraponera clavata. Venom samples were collected from a single colony either by manual venom gland dissection or by electrical stimulation and were compared using proteomic methods. Venom proteins were separated by 2D-PAGE and identified by nanoLC-ESI-QTOF MS/MS. Venom peptides were initially separated using C18 reversed-phase high performance liquid chromatography then analysed by MALDI-TOF MS. The proteomic analysis revealed numerous proteins that could be assigned a biological function (total 94) mainly as toxins, or roles in cell regulation and transport. This investigation found that ca. $73 \%$ of the proteins were common to venoms collected by the two methods. The peptidomic analysis revealed a large number of peptides (total 309 ) but with $<20 \%$ shared by the two collection methods. There was also a marked difference between venoms obtained by venom gland dissection from different ant colonies. These findings demonstrate the rich composition and variability of $P$. clavata venom.
\end{abstract}




\section{Introduction}

Ants of the order Hymenoptera are a diverse group of insects, with ca. 16,000 extant species belonging to 3 clades and 16 subfamilies ${ }^{1,2}$. The number of species within each subfamily ranges from 1 to more than $6000^{2}$. Of these subfamilies, 13 comprise ants that employ a stinger to inject a peptide-rich venom used for predation, defence and communication ${ }^{3-6}$. Although hundreds of peptides and proteins, in each venom, bear those functions, little work has been undertaken to characterise these components in detail (for recent reviews see $^{2,7}$ ).

Venom proteins and peptides are now routinely being investigated as drug or biopesticide leads, particularly those from bees ${ }^{8}$, spiders 9,10 and cone snails ${ }^{11-14}$ as many of these organisms are of medical importance, which suggests interesting pharmacological properties. Paraponera clavata (commonly known as the bullet ant or giant tropical ant) is a stinging ant that belongs to the subfamily Paraponerinae of the poneroid clade ${ }^{15,16}$. It is one of the few ants of medical importance due to its extremely painful sting ${ }^{16-18}$ that is used to deter predators and capture prey ${ }^{19}$. P. clavata venom is toxic to both vertebrates and invertebrates and is thought to elicit at least part of its action through the neurotoxic peptide poneratoxin ${ }^{20-23}$. This toxin induces slowly activating Nav1.7 mediated currents at low activation voltages and also sustained sodium currents as a result of a slowing of $\mathrm{Nav}$ channel inactivation ${ }^{16}$, similar to that described previously in rat and frog skeletal muscle ${ }^{23}$. All studies performed on this ant thus far have focussed on this peptide, as it is seen as a potential peptide for commercialisation as an insecticide due to its high activity ${ }^{20}$. An example of other ant-venom derived peptides that have been thoroughly investigated are ponericins from the ant Neoponera goeldii (Ponerinae; formerly Pachycondyla goeldii). These were found to have antimicrobial and insecticidal activities ${ }^{24}$. These peptides confirm that the venom of ants can be potential source of bioactive peptide leads that can be developed into therapeutic or insecticidal drugs.

To date the major hurdle for studying ant venoms has been the limited amount of venom present in their venom glands, however, this restriction has been overcome with recent advances in mass spectrometry and DNA sequencing techniques ${ }^{25}$. Ant venom is conventionally obtained by venom gland dissection, however it can also be collected by 
electrical stimulation, which can be considered a more efficient method of collection since ants remain alive following venom collection. These two collection methods have been employed with spider ${ }^{26}$, cone snail ${ }^{13}$, wasp ${ }^{27}$, scorpion ${ }^{28}$ and bee venoms ${ }^{3}$ and differences in the venom components have been seen between these two collection methods ${ }^{29-31}$. The dissection technique has been used for many years for a variety of different arthropods after it was first introduced in $1961{ }^{32}$. However, if isolation of only one certain venom component is desired, then electrical stimulation would be the better collection method, as it increases the likelihood of isolating the potent component due to the absence of contaminants such as structural proteins from the venom gland ${ }^{27,33}$. The other advantage of electrical stimulation, if performed en mass, is that it can give a higher yield of venom, is

less time consuming and keeps the organism alive for subsequent venom extraction ${ }^{34}$. If mass milking is not possible, as is the case with vespid venoms ${ }^{27}$, then automated methods of milking individual venoms have also been proposed ${ }^{35}$. Previous work suggests that dissected venom contains most of the proteins that are in electrically stimulated venom but also other proteins that are usually contaminants from the venom gland including cellular proteins used in metabolic machinery or toxin maturation and processing $13,27,36,37$. Electrically stimulated venom may therefore give a more genuine representation of the venom components. The present study aimed to compare the proteomic and peptidomic components of P. clavata obtained by electrical stimulation and manual dissection. While previous studies have looked at individual components of either the proteome or peptidome of ant venoms, this is the first in-depth study of both proteomic and peptidomic components. It also aimed to identify the presence of inter-colony variations to further confirm the diversity of this group of insects.

\section{Experimental Section}

\section{Venom Collection}

Venom samples from $P$. clavata were collected from two separate ant colonies at "la Montagne des Singes" near Kourou, French Guiana. To determine differences in venom profiles using the two collection procedures, ants from one colony (colony 1 ) were separated into two random groups and venom was extracted by either electrical stimulation or manual dissection of individual workers. To establish the extent of inter-colony variations 
in P. clavata venom profiles, venom from another colony (colony 2) was also obtained by venom gland dissection only. The manually dissected venom gland samples were prepared by storing ants at $-20^{\circ} \mathrm{C}$ prior to the dissection of the venom glands. After dissection, the glands were pooled in $10 \%(\mathrm{v} / \mathrm{v})$ acetonitrile $(\mathrm{ACN}) /$ water. Samples were then centrifuged for $5 \mathrm{~min}$ at $14,400 \mathrm{rpm}(12,000 \mathrm{gav})$, the supernatant was collected and lyophilized prior to storage at $-20^{\circ} \mathrm{C}$. Electrically stimulated venom was collected by placing individual $P$. clavata ants into a glass insert. Ants were then milked by placing a pair of tweezers attached to electrodes on their abdomen and a $12 \mathrm{~V}$ square wave pulse of $1 \mathrm{~ms}$ duration delivered at $100 \mathrm{~Hz}$. The venom was collected into a glass tube insert and diluted with $100 \mu \mathrm{L}$ of $10 \%$ ACN. The venom was then transferred to an Eppendorf tube prior to being freeze dried.

\section{Reverse-Phase High Performance Liquid Chromatography (RP-HPLC)}

Lyophilized crude electrically stimulated or manually dissected venom was separated on a Shimadzu HPLC system (Shimadzu, Kyoto, Japan) using a Vydac analytical C18 RP-HPLC column (218TP54, $25 \mathrm{~cm} \times 4.6 \mathrm{~mm}, 5 \mu \mathrm{m}$ particle size; Grace, MD, USA). Fractionation of venom peptide components was achieved using a gradient of $0.085 \%(\mathrm{v} / \mathrm{v})$ trifluoroacetic acid (TFA) in ACN (solvent B) in $0.1 \%(v / v)$ TFA in water (solvent A). Separation was achieved using a flow rate of $1 \mathrm{ml} / \mathrm{min}$ with the following gradient: $0-5 \mathrm{mins}, 0 \%$ solvent $\mathrm{B} ; 5-65 \mathrm{mins}$, 0-60\% B; 65-75 mins, 60-90\% B; 75-80 mins, 90\% B; 80-85 mins, 90-0\% B; and 85-90 mins, $0 \%$ B. Peaks were monitored at absorbances of $280 \mathrm{~nm}$ and $215 \mathrm{~nm}$ and manually collected, then fractions were lyophilised and stored at $-20^{\circ} \mathrm{C}$ for further analysis.

\section{MALDI-TOF MS Analysis}

MS analysis of each HPLC fraction was performed on an AB SCIEX TOF/TOF ${ }^{\mathrm{TM}} 5800$ mass spectrometer (AB SCIEX, MA, USA). Each venom fraction was manually collected and freezedried, then reconstituted in $10 \mu \mathrm{l}$ water. Subsequently $1 \mu \mathrm{l}$ of each fraction was then spotted onto a MALDI plate and overlaid with $0.5-0.75 \mu$ l of matrix using the dried droplet method 38. Matrix consisted of $5 \mathrm{mg} / \mathrm{ml}$ of $\alpha$-cyano-4-hydroxycinnamic acid (CHCA) dissolved in $1 \%$ (v/v) TFA / 100\% (v/v) ACN / $0.1 \mathrm{M} \mathrm{NH}_{4} \mathrm{H}_{2} \mathrm{PO}_{4}$ (45:45:10). Each spectrum was calibrated externally using a mixture of peptides with known molecular masses in the same $\mathrm{m} / \mathrm{z}$ range (AB SCIEX). Mass spectra were acquired in positive linear mode over the range 1,000-10,000 $\mathrm{m} / \mathrm{z}$ with 400 laser shots accumulated for each sample, based on the acceptance parameters 
and adequate signal intensity. Signals below $1,000 \mathrm{~m} / \mathrm{z}$ were not recorded as they are comprised of mostly matrix-related ion clusters ${ }^{39}$. Data Explorer ${ }^{\circledR}$ v4.11 software (AB SCIEX) was used to analyse spectra from individual RP-HPLC fractions to characterise the number and masses of peptides per venom, as previously reported ${ }^{9}$. Briefly, spectra were subjected to baseline correction with a correlation factor of 0.7 and Gaussian smoothing to reduce noise with a 5-point filter width. All mass attributions were verified manually and a mass list created for each LC-MALDI-TOF MS run. Potential adducts from oxidation, hydration, sodium, and deamination were manually removed from all mass lists, as well as any potential dimers or doubly-charged species. Peptides with mass matches within $\pm 1.0 \mathrm{Da}$ in adjoining HPLC fractions were considered identical and were removed from the data set as they likely reflect incomplete separation. For each venom, the peptides from each RP-HPLC fraction were consolidated into one mass list. Mass matching across data sets was performed in Microsoft Excel (Redmond, USA) to identify similar peptides across the different venoms. Two-dimensional scatter plots, termed "2D venom landscapes", were constructed using Prism v6 software (GraphPad, La Jolla, CA USA). All peptide masses detected in each HPLC fraction were plotted as a function of their $m / z$ values ( $x$-axis) and their HPLC retention time reflecting their hydrophobicity ( $y$-axis). Area-proportional Euler plots depicting overlapping peptide masses or proteins from different venoms were constructed using eulerAPE software (www.eulerdiagrams.org/eulerAPE/).

\section{Two-Dimensional Polyacrylamide Gel Electrophoresis (2D-PAGE)}

$300 \mu \mathrm{g}$ samples of venom, collected by electrical stimulation or manual dissection, were resuspended in $100 \mu \mathrm{l} 7 \mathrm{M}$ urea, $2 \mathrm{M}$ thiourea, 1\% (v/v) C7BzO, $50 \mathrm{mM}$ Tris $\mathrm{HCl}$ pH 8.8 before reduction and alkylation of disulphide bonds with $5 \mathrm{mM}$ tributylphosphine (TBP) and $20 \mathrm{mM}$ acrylamide monomers for 90 mins. The sample was subjected to 2D-PAGE as previously described ${ }^{40}$. Briefly, sample was fractionated by isoelectric focusing on an $11 \mathrm{~cm} \mathrm{pH} \mathrm{3-10}$ IPG strip (Bio-Rad, Hercules CA) for $100 \mathrm{kVh}$ and then further separated by molecular size by SDS-PAGE on a 4-20\% Tris-glycine gel (Criterion TGX, Bio-Rad, NSW, Australia). The gel was then placed in a fixing solution containing $40 \%(\mathrm{v} / \mathrm{v})$ methanol and $10 \%(\mathrm{v} / \mathrm{v})$ acetic acid for 30 mins at room temperature before staining with Flamingo fluorescent stain (Bio-Rad) for 1 h. After staining, the gel was scanned using a Molecular Imager PharosFX Plus system (BioRad) with QuantityOne ${ }^{\circledR}$ software. In order to perform nanoLC-ESI-QTOF MS/MS, gels were 
additionally stained with Coomassie Blue G250 overnight then destained with $1 \%(\mathrm{v} / \mathrm{v})$ acetic acid for $2 \mathrm{~h}$.

\section{NanoLC-ESI-QTOF MS/MS Analysis}

Gel spots or bands were excised and digested as previously described ${ }^{41}$. Peptide samples were then fractionated using a Tempo nano LC system (Eksigent, California, USA). Samples were first loaded onto a Michrom reversed-phase trapping cartridge at a rate of $20 \mu \mathrm{l} / \mathrm{min}$, then eluted onto a $75 \mu \mathrm{m} \times 150 \mathrm{~mm}$ PicoFrit column (New Objective, MA, USA) packed with Magic C18AQ chromatography resin (Bruker-Michrom Biosciences, CA, USA). An increasing gradient of ACN at $300 \mathrm{nl} / \mathrm{min}$ eluted the peptides which were ionised at $2300 \mathrm{~V}$ by the Microlonspray II head holding the PicoFrit column with integrated emitter into the source of a QSTAR Elite ${ }^{\mathrm{TM}}$ Quadrupole TOF mass spectrometer (AB SCIEX, MA, USA). An Intelligent Data Acquisition (IDA) experiment was performed, with a mass range of 350-1500 Da scanned for peptides of charge state $2^{+}$to $5^{+}$with an intensity of more than 30 counts/scan. The selected peptides were then fragmented, and the ion fragment masses were measured over a mass range of 50-1,500 Da. The mass of the precursor peptide was then excluded for $15 \mathrm{~s}$.

\section{Protein Identification}

The MS/MS data files were searched using Mascot (v2.4.0) against the LudwigNR database. This database comprised of the UniProt, plasmoDB and Ensembl databases (vQ114), provided by the Australian Proteomics Analysis Facility (APAF Ltd), hosted by the Walter and Eliza Hall Institute for Medical Research Systems Biology Mascot Server. The database was searched with the following parameter settings: fixed modifications, none; variable modifications, propionamide, oxidised methionine, deamidated asparagine and glutamine; enzyme, semi-trypsin; number of allowed missed cleavages, 3; peptide mass tolerance, 100 ppm; MS/MS mass tolerance, $0.2 \mathrm{Da}$; charge state, $2^{+}, 3^{+}$and $4^{+}$. PEAKS 7 software (Bioinformatics Solutions Inc., ON, Canada) was used to generate de novo sequences. A database comprising ant protein sequences available on the NCBI database and known contaminant proteins was compiled and uploaded onto PEAKS. De novo sequences were then also searched against this database. Remaining unassigned de novo sequences were searched on the online BLAST search engine MS-BLAST 
(http://genetics.bwh.harvard.edu/msblast/) ${ }^{42}$. Peptide identification used a cutoff score of $>20(-10 \lg P)$. Proteins were assigned as homologous when spots were located at the same position (mass and $\mathrm{p} /$ ) on at least two 2D gels and the protein was identified from the peptide fragment in at least one of the gel spots. Functions were assigned according to the GO annotation within UniProt, where available. Spectra have been provided for proteins matched with a coverage of less than $2 \%$ and have been included in the supporting information file (Figure S1-S4).

\section{Chemical Reagents}

All chemicals used were of analytical grade and, unless otherwise stated, were sourced from Sigma Aldrich (NSW, Australia). All buffers were prepared using Milli-Q (18 $\left.\mathrm{M} \Omega / \mathrm{cm}^{2}\right)$ water.

\section{Results}

\section{Peptidome Analysis}

Initially, the venom collected from P. clavata obtained by electrical stimulation and venom gland dissection of colony 1 was subjected to MALDI-TOF MS analysis. Venom obtained by either method was dominated by peptides in the mass range $2600-3200 \mathrm{~m} / \mathrm{z}$ (Figure 1AaBa). It was also found that no peptides appeared to be present at masses greater than 3200 $\mathrm{m} / \mathrm{z}$ and that manually dissected venom appeared to contain more peptides than electrically stimulated venom, particularly at masses $<2100 \mathrm{~m} / \mathrm{z}$. Nevertheless, the most intense peptide signal in both electrically stimulated and manually dissected venoms was of mass 2786.3 and $2787.6 \mathrm{~m} / \mathrm{z}$, respectively (Figure $1 \mathrm{Ab}-\mathrm{Bb}$ ).

Ants from colony 2 were also dissected and their venom glands pooled and analysed alongside those from colony 1 (Figure $1 \mathrm{Ca}$ ). The MALDI-TOF MS spectrum of the manually dissected colony 2 venom sample was similar to the one obtained from colony 1 , particularly the dissected venom sample. However, colony 2 venom had differences in the relative intensities of the major peptides as well as the presence of numerous additional peptides below $1500 \mathrm{~m} / \mathrm{z}$ (Figure 1Cb). Nevertheless, the most abundant peptide $2786.4 \mathrm{~m} / \mathrm{z}$ matched that seen in colony 1 samples $( \pm 1 \mathrm{Da})$. 
In order to reduce the complexity of the venoms, and therefore diminish the effects of ion suppression, all venoms were subsequently separated by C18 RP-HPLC. The HPLC chromatograms of all three venoms are shown in Figure $2 \mathrm{~A}-\mathrm{C}$. It can be seen that the venoms obtained by venom gland dissection and electrical stimulation from the same colony (colony 1 ) have similar profiles, each with 39 fractions. However, while the venom elution profile from colony 2 has many similar peaks, overall it has a different profile with only 29 peaks and an additional dominant peak at 50 min (Figure 2C).

Although each RP-HPLC fraction was seemingly homogenous, mostly with clear sharp peaks, multiple peptides were observed to co-elute in the same LC fraction when further resolved by MALDI-TOF MS analysis. This varied from no peptides in some fractions up to 32 masses in fraction 39 of the dissected venom. Overall, each venom contained a large number of small mass peptides, with cumulative total counts of 115 masses for electrically stimulated venom from colony 1, 137 masses for manually dissected venom from colony 1 and 354 for manually dissected venom from colony 2 . This highlights the overall complexity of $P$. clavata venom particularly from colony 2 . Although both dissected venoms had a similar mass distribution, with $95 \%$ of peptides < $2.9 \mathrm{kDa}$ (grey shaded areas in Figures 3B-C), the electrically stimulated venom from colony 1 contained a small number ( $n=15 ; 13 \%$ ) of higher mass peptides in the range 3-10 kDa that were not present in either of the dissected venoms from colony 1 or 2 (Figure 3A). Venom from colony 2 also had some similar, but not identical, high mass peptides, however they had a different RP-HPLC retention time compared to those from electrically stimulated venom from colony 1 , likely representing unique peptides. This further highlights the complexity of venoms obtained from different colonies of $P$. clavata.

Although there were only subtle differences in the chromatograms and the peptide mass distributions of the three venoms, comparisons between the peptide mass lists from the three venoms revealed large differences between venoms from each of the two collection methods and also between different colonies. Figure 4 shows that venoms obtained by the two different collection methods from ants of colony 1 had only 22 common masses between them (Figure 4C-D). This means that $81 \%$ and $84 \%$ are peptides unique to electrically stimulated and manually dissected venoms, respectively, despite the fact that the ants belong to the same colony. 


\section{Proteome analysis}

2D-PAGE analysis was performed in triplicate for each of the three samples, with representative gels shown in Figure 5. Overall the resultant gels look similar to one another, however when comparing the samples from the same colony (panels A and B of Figure 5), 24 spots were common (by mass and $\mathrm{p} /$ ) to both the electrically stimulated venom (total of 53 spots; Figure 5A) and the manually dissected venom (total of 38 spots; Figure 5B) gels. Interestingly, the sample from colony 2 (Figure 5C) contained no unique spots that were not present in either of the other two samples, and was not subjected to further analysis.

In order to identify whether the different collection methods resulted in different venom protein profiles, nanoLC-ESI-QTOF MS/MS analysis was performed on the 2D gel spots from P. clavata venom obtained from colony 1 by the different extraction techniques. Due to the absence of any specific proteomic or transcriptomic data for $P$. clavata, several different databases were searched in order to determine the highest homology matches. Two search engines were employed, Mascot and Peaks, while MS Blast was used to determine proteins with high homology to peptide sequences determined by de novo sequencing of MS/MS spectra. An extensive list of protein matches from each spot is included as Supplementary Tables S1 and S2. A total of 73 and 75 proteins were identified from venom obtained by electrical stimulation and manual dissection, respectively (Figure 6A), of which 54 identified proteins were common to both venoms (Figure 6B). This showed that although there were proteins shared by the two collection methods (Table S3), there still were identified proteins unique to each method: 21 for electrically stimulated venom and 19 for manually dissected venom.

In the $2 \mathrm{D}$ gels (Figure 5 ) it can be seen that the most abundant proteins in all three gels was a train of spots (\#12, \#40-42, and \#72) at ca. 18-21 kDa with an acidic p/ of ca. 4.5-5.5. These spots were all identified as isoforms of the enzyme phospholipase $A_{2}\left(P L A_{2}\right)$ in all three venom samples. PLA 2 proteins have been identified in many other hymenopteran venoms ${ }^{3,43-46}$. The high abundance of these proteins, particularly in manually dissected venom from colony 1 (Figure 5B), and the necessity not to overdevelop the gel may have resulted in less abundant proteins being below the limit of detection, particularly with Figure 5B. 
Other proteins that were common to all three venoms were spots \#5, \#25 and \#26. MS/MS analysis of spot \#5, located at ca. $51 \mathrm{kDa}$, with a p/ of ca. 5-6.5, revealed a match to the plasma glutamate carboxypeptidase, a secreted protein involved in peptide metabolism, in both spots. This protein has a reported mass of $53 \mathrm{kDa}$ and $\mathrm{p} /$ of 5.87 consistent with the spot's position. Spots \#25 and \#26 (ca. $25 \mathrm{kDa}, \mathrm{p} / 6.5$ ) both matched to the protein gammainterferon-inducible lysosomal thiol reductase, a $24 \mathrm{kDa}$ protein with a $\mathrm{p} /$ of 6.5 , involved in endosomal disulfide reduction.

As expected, Figure 6 shows that structural proteins are more prominent in manually dissected venom rather than electrically stimulated venom. For example, actin appeared at several spots in the manually dissected venom gel, including spots \#5, \#63 and \#74 (Figure 5B) at a mass of $36-38 \mathrm{kDa}$ and $\mathrm{p} /$ of ca. $5-7$, but only in an isolated spot \#8 from electrically stimulated venom (Figure 5A). Another example of a structural protein that was only found in manually dissected venom is flexible cuticle protein, a transmembrane protein, which was identified from spot \#69 (Figure 5B) and was not seen in the electrically stimulated venom.

In order to simplify this data, all protein matches were classified into six different functional categories: toxins, regulation, metabolism, structural, transport and undetermined. Figure $6 \mathrm{~A}$ shows that the two different collection methods result in venoms that contain functionally similar proteins. The main differences were seen in the structural and undetermined protein categories. The electrically stimulated venom had less structural and more undetermined proteins compared to the manually dissected venom (Figure 6A).

As seen in Fig. 6B and Table S3, each of the functional categories also had a high proportion of homologous proteins. For example, a regulatory protein identified from spot \#19 in both gels was the $26 \mathrm{~S}$ proteasome non-ATPase regulatory subunit 1 Tables S1 - S3. While an example of a toxin/allergen protein present in both venoms was $\mathrm{PLA}_{2}$, which was seen in many spots such as in spots \#12, \#14, \#42 and \#43 (Tables S1 - S3). Another toxin identified was allergen sol i $1 \mathrm{II}^{47}$, however it was only seen in spot \#37 of the electrically stimulated venom.

\section{Discussion}


Venom collection is the rate-limiting step in venomic studies of small animals and restricts further investigation for drug and bioinsecticide discovery, allergenicity studies and antivenom development ${ }^{2}$. The two main methods of collecting venoms are either electrostimulation or manual dissection of the venom gland ${ }^{48}$, with the obvious disadvantage of the latter procedure being that the animal must be sacrificed. The present investigation used proteomic and peptidomic techniques to investigate the difference between these two collection methods and also identified differences in venom composition between different $P$. clavata ant colonies. While these two venom collection methods have previously been employed to investigate venom composition from other organisms such as bees ${ }^{3}$, cone snails ${ }^{13}$ and spiders ${ }^{25}$, the present study represents the first systematic investigation of ant venoms obtained using these two methods. Both electrical stimulation 5, 49 and manual dissection ${ }^{50,51}$ collection methods for ant venoms have been used in separate studies; however, any differences between these two methods were not established. The present investigation represents the first in-depth systematic study of both the peptidome and proteome from a single species of ant and is the first comprehensive study of $P$. clavata venom, an ant that causes one of the most painful insect sting ${ }^{48}$. Whilst there was no major disparity in the numbers of peptides and proteins in venoms collected using the two methods, closer investigation revealed that there were distinct differences in the masses of these peptides and the types of proteins.

\section{Variations in Peptidome}

The two collection methods were first compared based on the MALDI-TOF mass profiles of electrically stimulated versus manually dissected whole venom. P. clavata venom was found to be rich in peptides with no major differences between venom collection techniques. We therefore performed C18 RP-HPLC separation of the two venoms followed by offline analysis of the chromatographic fractions (LC-MALDI-TOF MS). This was to avoid the ion suppression effects known to occur in MALDI-TOF MS with complex venoms such as spiders, cone snails and both poneroid and formicoid ants 9, 52. The RP-HPLC chromatograms were not significantly different and the large number and mass distribution of the peptides below 4 kDa was similar to that reported for other poneroid ant venoms ${ }^{51-54}$. This mass range of peptides is not unique to ant venoms, as cone snail venom contains mostly low molecular weight peptides in their venoms ${ }^{55}$. Nevertheless, other venomous organisms such as spiders 
and scorpions have peptides in the higher molecular weight range of approximately 3-10 $\mathrm{kDa}{ }^{9,56}$. However, comparison of individual masses within ant venom LC fractions found that each collection method resulted in venoms with unique peptide masses. As expected, there were more peptides unique to manually dissected venom compared to electrically stimulated venom (42 vs. 31), probably reflecting peptides, or products of proteins, that are part of the venom gland itself or are part of the gland's machinery for toxin maturation and processing ${ }^{13,25}$. Other reasons for this high variability might be attributed to the pooled ants' having different diets ${ }^{57}$ or being in different stages of development which may give rise to ontogenic variation as shown in several venomous animals such as wasps ${ }^{58}$, spiders ${ }^{59}$ and snakes ${ }^{60}$. The process of electrical stimulation of the abdomen may not only release peptides from the venom gland, but also peptides and breakdown products of other glands. These may include antimicrobial and antifungal peptide contaminants from the digestive tract or hemolymph ${ }^{61}$ and peptides and proteins that are breakdown products involved in the biosynthesis of trail pheromones and other exocrine secretions.

Previous work on the ant $P$. clavata has been focussed on poneratoxin (PoTx), a neurotoxic

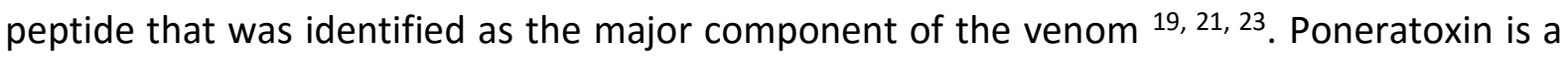
25-residue peptide with an average mass of 2756.4 Da that has been investigated for its potential development as a bioinsecticide ${ }^{19,20,62}$. More recently an investigation by Johnson et al. (2016) identified three new isoforms of poneratoxin with average masses of 2814.4 Da (glyceryl-PoTx; the presumed precursor of PoTx with an additional glycine residue at position 26), 2814.4 Da (A23E PoTx) and 2783.5 Da (D22N A23V PoTx) that were isolated from ants collected from different geographical locations. Post-translational modifications to peptides are not unique to $P$. clavata venom, but are common in many animal venoms such as other ants ${ }^{53,63}$, wasps ${ }^{64}$, spiders ${ }^{65}$ and especially marine cone snails ${ }^{53}$. These modifications have been attributed to the high peptide counts seen in these venoms as well as the variations between species ${ }^{29}$. In support of Johnson et al (2016) we also identified a peptide of mass ca. 2784.4 Da as the most dominant peptide in venoms obtained by the two collection methods from colony 1 and also from venom from colony 2 (see Figure 2 for elution times). Based on mass, we were only able to identify PoTx from venom obtained from colony 2. This further supports the idea that there are several poneratoxin homologs in P. clavata geographical variants and within venom from a single colony. 


\section{Variations in Proteome}

The $P$. clavata proteome seems to be on a similar level of complexity, in terms of spot numbers, as other hymenopteran venoms ${ }^{3,66}$ and other poneroid ants ${ }^{52,67}$. However, $P$. clavata venom is less complex, in terms of protein numbers and mass ranges, to that of other venomous organisms such as cone snails ${ }^{68}$, snakes ${ }^{69,} 70$ and spiders which have up to 300 protein spots $^{69}$. Using a 2D-PAGE approach, there was also a large number of protein spots that were shared between the two collection methods, for example, spots \#54 and \#39 were resolved in the same position (mass and $\mathrm{p} /$ ) in both electrically stimulated and manually dissected venoms. In contrast, there were several spots that were unique to each collection method: spot \#29 in electrically stimulated venom and \#14 manually dissected venom. While manually dissected venom was expected to have more proteins due to likely contamination of venom gland proteins ${ }^{3,29}$, a number of high abundance proteins may have obscured low abundance ones ${ }^{66}$. This included a number of high intensity spots on the acidic end of the manually dissected venom gel at approximately $20 \mathrm{kDa}$, later identified as $\mathrm{PLA}_{2}$ homologues.

nanoLC-ESI-QTOF MS-MS analysis of 2D gel spots identified several proteins within the venom using both collection methods. The most abundant proteins from electrically stimulated and manually dissected venoms were identified as $\mathrm{PLA}_{2}$ homologues. This is a common venom toxin reported in wasp ${ }^{71}$, bee ${ }^{3}$, snake ${ }^{74}$ and ant $44,52,72$ venom as well as being a major allergen in hymenopteran ${ }^{73}$ venoms. Its activity involves disruption of phospholipid membranes leading to pore formation, cell lysis, cardiac dysfunction and ultimately death of the organism ${ }^{71}$. Other stinging hymenoptera have also been found to have high levels of $\mathrm{PLA}_{2}$ that account for at least some their toxic activity ${ }^{75}$. For example, $12 \%$ of the dry weight of bumblebee venom are $\mathrm{PLA}_{2} \mathrm{~S}{ }^{75}$. The lipase property of phospholipases may also facilitate further spreading of venom through the host tissue ${ }^{13}$. The allergen sol i III, originally identified in the venom of the ant Solenopsis invicta, was also present in $P$. clavata venom, and may contribute to the overall toxicity, particularly allergic responses ${ }^{47}$ despite these have not been widely reported with $P$. clavata envenomation. As there were no other proteins with potential toxic activity identified, it appears that the toxic effects of $P$. clavata venom are the result of poneratoxin as suggested by Johnson ${ }^{16}$, perhaps in combination with PLA 2 isoforms. Nevertheless, the sting by $P$. clavata does not 
just involve a very painful sting, but also other systemic effects such as haemolysis ${ }^{76}$ and tonic-clonic seizures that have been observed in mice that ultimately lead to death at relatively low doses $(6 \mathrm{mg} / \mathrm{kg}){ }^{77}$. The venom components that cause these additional activities remain unidentified.

The 2D gels of $P$. clavata venom obtained in this study contained several spots that were identified as $\mathrm{PLA}_{2}$ proteins, an observation reported for other venoms, including cone snails, snakes and wasps $29,73,74$. Interestingly, the PLA 2 proteins have a range of different $p /$ values and masses. Variance in the $\mathrm{p} /$ of $\mathrm{PLA}_{2}$ homologues present in $P$. clavata venom can be explained by differences in the amino acid sequence of PLA $A_{2}$ subunits resulting in a range of basic and acidic PLA 2 proteins, as previously described in hymenopterans and snakes ${ }^{73}, 74$. The variability in mass can be explained by homology with multimers of PLA $A_{2}$ subunits (often with different individual subunit masses) or monomer PLA 2 proteins, also with varying degrees of glycosylation on individual subunits ${ }^{74}$. Despite the coverage scores of the matched proteins not being particularly high, it can be safely assumed that the protein matches are correct, as this enzyme is known to be highly conserved within Hymenoptera ${ }^{73}$ and has also been reported in $P$. clavata using biochemical assays 76,77 .

Although peptides perform a significant role in venom functions, proteins can act as carriers, neurotoxins or derivative enzymes such as proteases to help in the maturation and function of the peptides ${ }^{13}$. These proteins may also have synergistic effects on the toxic components of venoms ${ }^{65}$. Our study identified several manually dissected venom proteins that are located intracellularly, and we observed that electrically stimulated venom had fewer of these. In particular these were structural proteins such as actin and cellular transport proteins such as myosin. This has also been reported in previous work that compared these two techniques using other venoms such as that of bees and marine cone snails ${ }^{66,78}$. It has therefore been claimed that electrically stimulated venom is more representative of the injected venom compared to manually dissected venom ${ }^{3}$. The intracellular proteins are usually thought of as artefacts of dissection, however it has been proposed that they might be co-secreted into the venom gland along with a toxin ${ }^{29,65}$. In support, we saw some structural proteins in electrically stimulated venom as well. 
The relative proportion of proteins allocated to each category in this study was not markedly different to those of other venoms, with the majority characterised as intracellular proteins ${ }^{3,29}$. There was also a large number of proteins in the unknown category, a recurring issue in most venomics studies. This is due to the lack of genomic and transcriptomic bioinformatics data available for a number of venoms, especially those of ants, as well as the added problem of low abundance 'masking' due to high abundance proteins $25,65,79$.

\section{Inter-colony Variations}

There were several differences between the venom from ants of colony 1 to those of colony 2 in both the peptide and protein numbers and protein composition. This has been previously observed with other ant species such as Odontomachus haematodus ${ }^{80}$, Dinoponera quadriceps ${ }^{53}$ and other venomous organisms including marine cone snails ${ }^{11}$ and wasps ${ }^{64}$. The reason for these marked differences has been attributed to different factors such as age, size, differential gene expression, altered post-translational modifications and, in the case of ants, genetic polymorphisms that result from the queens mating with different males during their lifetime ${ }^{64,81}$. It has also been previously shown that although the organisms are from the same region, as was the case with these colonies, slight environmental differences between the two colonies may explain the differences seen $^{53,64}$. For example, for a predatory ant like $P$. clavata, prey diversity and abundance, as well as other factors such as peptide composition during venom regeneration and the overall health of individual ants may affect peptide levels and expression ${ }^{53}$.

\section{Concluding Remarks}

The present study has shown that venom obtained by manual venom gland dissection reveals a broader number of peptides and proteins than electrically stimulated $P$. clavata venom. From the 2D-PAGE analysis, approximately $72-74 \%$ of the proteins are shared by the two collection methods. It was found that the manually dissected venom contained a number of additional regulatory proteins, while the electrically stimulated venom had a number of undetermined proteins. However, there is no evidence suggesting that these additional undetermined and regulatory proteins are toxic. Unexpectedly, the overlap in peptide mass matches (approximately 16-19\%) was far less than what was observed from 
protein matches. Although extracting venom via electrical stimulation is the preferred method of collection, as it is not destructive to the ants and contains all the major protein components, this work collected electrically stimulated venom by individually milking ants which was not an efficient method, and future work should utilise a mass milking technique such as that described by Eskridge et al. (1981) ${ }^{82}$. It must be noted that the peptide composition differs markedly to that of manually dissected venom and both methods may need to be used to obtain a more accurate representation of venom contents. This venom complexity is further increased when venoms from different colonies are compared. This highlights the diversity of ant venom proteins and peptides that can be characterised between colonies, and by using different collection techniques, for the discovery of potential therapeutic or insecticidal leads.

\section{Supporting Information}

The following files are available free of charge at ACS website http://pubs.acs.org:

- Supplementary Table S1: Protein assignment of $P$. clavata dissected venom 2D-PAGE spots identified by nanoLC-ESI-QTOF MS/MS

- Supplementary Table S2: Protein assignment of $P$. clavata electrically stimulated venom 2D-PAGE spots identified by nanoLC-ESI-QTOF MS/MS

- Supplementary Table S3: $P$. clavata proteins identified from 2D-PAGE spots of electrically stimulated and manually dissected venom. Table shows proteins identified by each method as well as proteins unique to each collection method

- Supplementary Figure S1: Annotated MS spectra of peptides from manually dissected P. clavata 2D gel spot 22 used to match against hymenopteran proteins.

- Supplementary Figure S2: Annotated MS spectra of peptides from manually dissected P. clavata 2D gel spot 58 used to match against hymenopteran proteins.

- Supplementary Figure S3: Annotated MS spectra of peptides from electrically stimulated P. clavata 2D gel spot 26 used to match against hymenopteran proteins.

- Supplementary Figure S4: Annotated MS spectra of peptides from electrically stimulated P. clavata 2D gel spot 45 used to match against hymenopteran proteins.

\section{Acknowledgements}


Financial support for this study was provided by an Australian Postgraduate Award to Samira R. Aili and the programme convergence 2007-2013, Région Guyane from the European community (Bi-Appli, 115/SGAR-DE-2011/052274) to Alain Dejean. This work has also benefited from an "Investissement d'Avenir" grant managed by the Agence Nationale de la Recherche (CEBA, ref. ANR-10-LABX-25-01).

\section{Abbreviations}

ACN, acetonitrile; C7BzO, 3-(4-heptyl)phenyl-3-hydroxypropyl) dimethylammoniopropane sulfonate; 1,5-DAN, 1,5-diaminonapthalene; CHCA, $\alpha$-cyano-4-hydroxycinnamic acid; FA, ferulic acid; HGD, Hymenopteran Genome Database; IEF, isoelectric focussing; ionic CHCA, $\mathrm{N}$-tert-butyl-N-isopropyl-N-methylammonium $\quad \alpha$-cyano-4-hydroxycinnamate; IPG, immobilized pH gradient, LC-ESI-QTOF MS, liquid chromatography electrospray ionization quadrupole time-of-flight mass spectrometry; MALDI-TOF MS, matrix-assisted laserdesorption ionization time-of-flight mass spectrometry; PLA2, phospholipase A2; RP-HPLC, reverse-phase high pressure liquid chromatography; SDS-PAGE, sodium dodecyl sulphate polyacrylamide gel electrophoresis; TFA, trifluoroacetic acid.

\section{References}

1. AntWeb, Available online: http://www.antweb.org (accessed on 26 August 2016) 2014.

2. Aili, S. R.; Touchard, A.; Escoubas, P.; Padula, M. P.; Orivel, J.; Dejean, A.; Nicholson, G. M., Diversity of peptide toxins from stinging ant venoms. Toxicon 2014, 92, 166-78.

3. L Li, R.; Zhang, L.; Fang, Y.; Han, B.; Lu, X.; Zhou, T.; Feng, M.; Li, J., Proteome and phosphoproteome analysis of honeybee (Apis mellifera) venom collected from electrical stimulation and manual extraction of the venom gland. BMC Genomics 2013, 14, 766.

4. Ward, P. S., Taxonomy, phylogenetics, and evolution. In Ant ecology Lach R., P. C. L., Abbott K. L., , Ed. 2010; Vol. 1, pp 3-17.

5. Pluzhnikov, K. A.; Kozlov, S. A.; Vassilevski, A. A.; Vorontsova, O. V.; Feofanov, A. V.; Grishin, E. V., Linear antimicrobial peptides from Ectatomma quadridens ant venom. Biochimie 2014, $107 \mathrm{Pt}$ B, 211-5.

6. Touchard, A.; Koh, J.; Aili, S. R.; Dejean, A.; Nicholson, G. M.; Orivel, J.; Escoubas, P., The complexity and structural diversity of ant venom peptidomes is revealed by mass spectrometry profiling. Rapid Commun. Mass Spectrom. 2015, 29, (5), 385-96. 
7. Touchard, A.; Aili, S.; Fox, E.; Escoubas, P.; Orivel, J.; Nicholson, G.; Dejean, A., The biochemical toxin arsenal from ant venoms. Toxins (Basel) 2016, 8, (1), 30.

8. Wood, C. L.; Hoffman, D. R., Two-dimensional polyacrylamide gel electrophoresis of hymenoptera venom and venom sac extracts. Toxicon 1983, 21, (2), 291-9.

9. Palagi, A.; Koh, J. M.; Leblanc, M.; Wilson, D.; Dutertre, S.; King, G. F.; Nicholson, G. M.; Escoubas, P., Unravelling the complex venom landscapes of lethal Australian funnel-web spiders (Hexathelidae: Atracinae) using LC-MALDI-TOF mass spectrometry. J. Proteomics 2013, 80, 292-310. 10. Windley, M. J.; Herzig, V.; Dziemborowicz, S. A.; Hardy, M. C.; King, G. F.; Nicholson, G. M., Spider-venom peptides as bioinsecticides. Toxins (Basel) 2012, 4, (3), 191-227.

11. Dutertre, S.; Biass, D.; Stöcklin, R.; Favreau, P., Dramatic intraspecimen variations within the injected venom of Conus consors: An unsuspected contribution to venom diversity. Toxicon 2010, 55, (8), 1453-62.

12. Dutertre, S.; Jin, A. H.; Kaas, Q.; Jones, A.; Alewood, P. F.; Lewis, R. J., Deep venomics reveals the mechanism for expanded peptide diversity in cone snail venom. Mol. Cell. Proteomics 2013, 12, (2), 312-29.

13. Möller, C.; Vanderweit, N.; Bubis, J.; Marí, F., Comparative analysis of proteases in the injected and dissected venom of cone snail species. Toxicon 2013, 65, (0), 59-67.

14. Peigneur, S.; Van Der Haegen, A.; Möller, C.; Waelkens, E.; Diego-García, E.; Marí, F.; Naudé, R.; Tytgat, J., Unraveling the peptidome of the South African cone snails Conus pictus and Conus natalis. Peptides 2013, 41, 8-16.

15. Larson, H. K.; Goffredi, S. K.; Parra, E. L.; Vargas, O.; Pinto-Tomas, A. A.; McGlynn, T. P., Distribution and dietary regulation of an associated facultative Rhizobiales-related bacterium in the omnivorous giant tropical ant, Paraponera clavata. Naturwissenschaften 2014, 101, (5), 397-406.

16. Johnson, S. R.; Rikli, H. G.; Schmidt, J. O.; Evans, M. S., A reexamination of poneratoxin from the venom of the bullet ant Paraponera clavata. Peptides 2016, (In Press).

17. Bosmia, A. N.; Griessenauer, C. J.; Haddad, V.; Shane Tubbs, R., Ritualistic envenomation by bullet ants among the Sateré-Mawé Indians in the Brazilian Amazon. Wilderness Environ. Med. 2015, 26, (2), 271-273.

18. Torres, A.; Quinet, Y.; Havt, A.; Rádis-Baptista, G.; Martins, A., Molecular pharmacology and toxynology of venom from ants. An Integrated view of the molecular recognition and toxinologyFrom analytical procedures to biomedical applications. Croatia: InTech 2013, 207-22.

19. Piek, T.; Hue, B.; Mantel, P.; Nakajima, T.; Schmidt, J. O., Pharmacological characterization and chemical fractionation of the venom of the ponerine ant, Paraponera clavata (F.). Comparative Biochemistry and Physiology Part C: Comparative Pharmacology 1991, 99, (3), 481-6. 
20. Szolajska, E.; Poznanski, J.; Ferber, M. L.; Michalik, J.; Gout, E.; Fender, P.; Bailly, I.; Dublet, B.; Chroboczek, J., Poneratoxin, a neurotoxin from ant venom. Structure and expression in insect cells and construction of a bio-insecticide. Eur. J. Biochem. 2004, 271, (11), 2127-36.

21. Piek, T.; Duval, A.; Hue, B.; Karst, H.; Lapied, B.; Mantel, P.; Nakajima, T.; Pelhate, M.; Schmidt, J. O., Poneratoxin, a novel peptide neurotoxin from the venom of the ant, Paraponera clavata. Comp. Biochem. Physiol., C: Comp. Pharmacol. 1991, 99, (3), 487-95.

22. Piek, T., Neurotoxins from venoms of the Hymenoptera--twenty-five years of research in Amsterdam. Comp. Biochem. Physiol. C 1990, 96, (2), 223-33.

23. Duval, A.; Malécot, C. O.; Pelhate, M.; Piek, T., Poneratoxin, a new toxin from an ant venom, reveals an interconversion between two gating modes of the Na channels in frog skeletal muscle fibres. Pflügers Archiv 1992, 420, (3-4), 239-47.

24. Orivel, J.; Redeker, V.; Le Caer, J. P.; Krier, F.; Revol-Junelles, A. M.; Longeon, A.; Chaffotte, A.; Dejean, A.; Rossier, J., Ponericins, new antibacterial and insecticidal peptides from the venom of the ant Pachycondyla goeldii. J. Biol. Chem. 2001, 276, (21), 17823-29.

25. Garb, J. E., Extraction of venom and venom gland microdissections from spiders for proteomic and transcriptomic analyses. J Vis Exp 2014, (93), e51618.

26. da Silveira, R. B.; Chaim, O. M.; Mangili, O. C.; Gremski, W.; Dietrich, C. P.; Nader, H. B.; Veiga, S. S., Hyaluronidases in Loxosceles intermedia (Brown spider) venom are endo- $\beta-\mathrm{N}$-acetyl-dhexosaminidases hydrolases. Toxicon $\mathbf{2 0 0 7 , 4 9 , ( 6 ) , 7 5 8 - 7 6 8 . ~}$

27. Mueller, U.; Reisman, R.; Wypych, J.; Elliott, W.; Steger, R.; Walsh, S.; Arbesman, C., Comparison of vespid venoms collected by electrostimulation and by venom sac extraction. J. Allergy Clin. Immunol. 1981, 68, (4), 254-61.

28. Inceoglu, B.; Lango, J.; Jing, J.; Chen, L.; Doymaz, F.; Pessah, I. N.; Hammock, B. D., One scorpion, two venoms: Prevenom of Parabuthus transvaalicus acts as an alternative type of venom with distinct mechanism of action. Proc. Natl. Acad. Sci. U. S. A. 2003, 100, (3), 922-927.

29. Leonardi, A.; Biass, D.; Kordiš, D.; Stöcklin, R.; Favreau, P.; Križaj, I., Conus consors snail venom proteomics proposes functions, pathways, and novel families involved in its venomic system. J. Proteome Res. 2012, 11, (10), 5046-58.

30. Gopalakrishnakone, P.; Cheah, J.; Gwee, M. C., Black scorpion (Heterometrus longimanus) as a laboratory animal: maintenance of a colony of scorpion for milking of venom for research, using a restraining device. Lab. Anim. 1995, 29, (4), 456-8.

31. Oukkache, N.; Chgoury, F.; Lalaoui, M.; Cano, A. A.; Ghalim, N., Comparison between two methods of scorpion venom milking in Morocco. J. Venom. Anim. Toxins Incl. Trop. Dis. 2013, 19, (1), 1-5. 
32. Shulman, S.; Langlois, C.; Arbesman, C. E., The allergic response to stinging insects: I. Preparation of extracts and their biochemical characterization. J. Allergy 1964, 35, (5), 446-63.

33. O'Connor, R.; Rosenbrook, W., Jr.; Erickson, R., Disc electrophoresis of hymenoptera venoms and body proteins Science 1964, 145, (3638), 1320-21.

34. Benton, A. W.; Morse, R. A.; Stewart, J. D., Venom collection from honey bees. Science 1963, 142, (3589), 228-30.

35. O'Connor, R.; Rosenbrook, W.; Erickson, R., Hymenoptera: pure venom from bees, wasps, and hornets. Science 1963, 139, (3553), 420.

36. Hsiang, H. K.; Elliott, W. B., Differences in honey bee (Apis mellifera) venom obtained by venom sac extraction and electrical milking. Toxicon 1975, 13, (2), 145-48.

37. Wood, C. L.; Timmons, B. E. t.; Hoffman, D. R., Allergens in hymenoptera venoms. X. Vespid venoms versus venom sac extracts: comparison by two-dimensional polyacrylamide gel electrophoresis. Ann. Allergy 1983, 51, (4), 441-45.

38. Jaskolla, T. W.; Karas, M.; Roth, U.; Steinert, K.; Menzel, C.; Reihs, K., Comparison between vacuum sublimed matrices and conventional dried droplet preparation in MALDI-TOF mass spectrometry. J. Am. Soc. Mass. Spectrom. 2009, 20, (6), 1104-14.

39. Escoubas, P.; Sollod, B.; King, G. F., Venom landscapes: mining the complexity of spider venoms via a combined cDNA and mass spectrometric approach. Toxicon 2006, 47, (6), 650-63. 40. Jobbins, S. E.; Hill, C. J.; D’Souza-Basseal, J. M.; Padula, M. P.; Herbert, B. R.; Krockenberger, M. B., Immunoproteomic approach to elucidating the pathogenesis of cryptococcosis caused by Cryptococcus gattii. J. Proteome Res. 2010, 9, (8), 3832-41.

41. Raymond, B. B. A.; Tacchi, J. L.; Jarocki, V. M.; Minion, F. C.; Padula, M. P.; Djordjevic, S. P., P159 from Mycoplasma hyopneumoniae binds porcine cilia and heparin and is cleaved in a manner akin to ectodomain shedding. J. Proteome Res. 2013, 12, (12), 5891-903.

42. Shevchenko, A.; Sunyaev, S.; Loboda, A.; Shevchenko, A.; Bork, P.; Ens, W.; Standing, K. G., Charting the proteomes of organisms with unsequenced genomes by MALDI-quadrupole time-offlight mass spectrometry and BLAST homology searching. Anal. Chem. 2001, 73, (9), 1917-26.

43. Hoffman, D. R., Allergens in bee venom: III. Identification of allergen B of bee venom as an acid phosphatase. J. Allergy Clin. Immunol. 1977, 59, (5), 364-66.

44. Hoffman, D. R.; Sakell, R. H.; Schmidt, M., Sol i 1, the phospholipase allergen of imported fire ant venom. J. Allergy Clin. Immunol. 2005, 115, (3), 611-16.

45. Lewis, J. C.; Day, A. J.; De la Lande, I. S., Phospholipase A in the venom of the Australian bulldog ant Myrmecia pyriformis. Toxicon 1968, 6, (2), 109-12. 
46. Zalat, S.; Schmidt, J.; Moawad, T. I., Lipase and phospholipase activities of Hymenoptera venoms (wasps and ants). Egyptian J Biol 2003, 5, 138-47.

47. Schmidt, M.; McConnell, T. J.; Hoffman, D. R., Immunologic characterization of the recombinant fire ant venom allergen Sol i 3 . Allergy 2003, 58, (4), 342-49.

48. Dossey, A., Chemical Defenses of Insects: A Rich Resource for Chemical Biology in the Tropics. In Chemical Biology of the Tropics, Vivanco, J. M.; Weir, T., Eds. Springer Berlin Heidelberg: 2011; Vol. 8, pp 27-57.

49. Hink, W. F.; Pappas, P. W.; Jaworski, D. C., Partial biochemical characterization of venom from the ant, Pseudomyrmex triplarinus. Toxicon 1994, 32, (7), 763-72.

50. Touchard, A.; Labrière, N.; Roux, O.; Petitclerc, F.; Orivel, J.; Escoubas, P.; Koh, J. M.; Nicholson, G. M.; Dejean, A., Venom toxicity and composition in three Pseudomyrmex ant species having different nesting modes. Toxicon 2014, 88, 67-76.

51. Touchard, A.; Dauvois, M.; Arguel, M.-J.; Petitclerc, F.; Leblanc, M.; Dejean, A.; Orivel, J.; Nicholson, G. M.; Escoubas, P., Elucidation of the unexplored biodiversity of ant venom peptidomes via MALDI-TOF mass spectrometry and its application for chemotaxonomy. J. Proteomics 2014, 105, 217-31.

52. Aili, S. R.; Touchard, A.; Koh, J. M. S.; Dejean, A.; Orivel, J.; Padula, M. P.; Escoubas, P.; Nicholson, G. M., Comparisons of protein and peptide complexity in poneroid and formicoid ant venoms. J. Proteome Res. 2016, 15, (9), 3039-54.

53. Cologna, C. T.; Cardoso Jdos, S.; Jourdan, E.; Degueldre, M.; Upert, G.; Gilles, N.; Uetanabaro, A. P.; Costa Neto, E. M.; Thonart, P.; de Pauw, E.; Quinton, L., Peptidomic comparison and characterization of the major components of the venom of the giant ant Dinoponera quadriceps collected in four different areas of Brazil. J. Proteomics 2013, 94, 413-22.

54. Johnson, S. R.; Copello, J. A.; Evans, M. S.; Suarez, A. V., A biochemical characterization of the major peptides from the venom of the giant neotropical hunting ant Dinoponera australis. Toxicon 2010, 55, (4), 702-10.

55. Davis, J.; Jones, A.; Lewis, R. J., Remarkable inter- and intra-species complexity of conotoxins revealed by LC/MS. Peptides 2009, 30, (7), 1222-27.

56. Abdel-Rahman, M. A.; Harrison, P. L.; Strong, P. N., Snapshots of scorpion venomics. J. Arid Environ. 2015, 112, 170-176.

57. Pucca, M. B.; Amorim, F. G.; Cerni, F. A.; Bordon, K. d. C. F.; Cardoso, I. A.; Anjolette, F. A. P.; Arantes, E. C., Influence of post-starvation extraction time and prey-specific diet in Tityus serrulatus scorpion venom composition and hyaluronidase activity. Toxicon 2014, 90, 326-36. 
58. Uçkan, F.; Ergin, E.; Rivers, D. B.; Gençer, N., Age and diet influence the composition of venom from the endoparasitic wasp Pimpla turionellae L.(Hymenoptera: Ichneumonidae). Arch. Insect Biochem. Physiol. 2006, 63, (4), 177-87.

59. Herzig, V.; Ward Rj Fau - Dos Santos, W. F.; Dos Santos, W. F., Ontogenetic changes in Phoneutria nigriventer (Araneae, Ctenidae) spider venom. Toxicon 2004, 44, (0041-0101 (Print)), 635-40.

60. Alape-Giron, A.; Sanz, L.; Escolano, J.; Flores-Diaz, M.; Madrigal, M.; Sasa, M.; Calvete, J. J., Snake venomics of the lancehead pitviper Bothrops asper: geographic, individual, and ontogenetic variations. J. Proteome Res. 2008, 7, (8), 3556-71.

61. Bulet, P.; Stocklin, R., Insect antimicrobial peptides: structures, properties and gene regulation. Protein and peptide letters 2005, 12, (1), 3-11.

62. Hendrich, A. B.; Mozrzymas, J. W.; Konopińska, D.; Scuka, M., The effect of poneratoxin on neuromuscular transmission in the rat diaphragm. Cell. Mol. Biol. Lett. 2001, 7, (2), 195-202.

63. Wiese, M. D.; Chataway, T. K.; Davies, N. W.; Milne, R. W.; Brown, S. G.; Gai, W. P.; Heddle, R. J., Proteomic analysis of Myrmecia pilosula (jack jumper) ant venom. Toxicon 2006, 47, (2), 20817.

64. Dias, N. B.; de Souza, B. M.; Gomes, P. C.; Palma, M. S., Peptide diversity in the venom of the social wasp Polybia paulista (Hymenoptera): a comparison of the intra- and inter-colony compositions. Peptides 2014, 51, 122-30.

65. Yuan, C.; Jin, Q.; Tang, X.; Hu, W.; Cao, R.; Yang, S.; Xiong, J.; Xie, C.; Xie, J.; Liang, S., Proteomic and peptidomic characterization of the venom from the Chinese bird spider, Ornithoctonus huwena Wang. J. Proteome Res. 2007, 6, (7), 2792-801.

66. Sookrung, N.; Wong-din-Dam, S.; Tungtrongchitr, A.; Reamtong, O.; Indrawattana, N.; Sakolvaree, Y.; Visitsunthorn, N.; Manuyakorn, W.; Chaicumpa, W., Proteome and allergenome of Asian wasp, Vespa affinis, venom and IgE reactivity of the venom components. J. Proteome Res. 2014, 13, (3), 1336-44.

67. Pessoa, W. F. B.; Silva, L. C. C.; de Oliveira Dias, L.; Delabie, J. H. C.; Costa, H.; Romano, C. C., Analysis of protein composition and bioactivity of Neoponera villosa venom (Hymenoptera: Formicidae). International journal of molecular sciences 2016, 17, (4), 513.

68. Safavi-Hemami, H.; Siero, W. A.; Gorasia, D. G.; Young, N. D.; MacMillan, D.; Williamson, N. A.; Purcell, A. W., Specialisation of the venom gland proteome in predatory cone snails reveals functional diversification of the conotoxin biosynthetic pathway. J. Proteome Res. 2011, 10, (9), 3904-3919. 
69. Igci, N.; Demiralp, D. O., A preliminary investigation into the venom proteome of Macrovipera lebetina obtusa (Dwigubsky, 1832) from Southeastern Anatolia by MALDI-TOF mass spectrometry and comparison of venom protein profiles with Macrovipera lebetina lebetina (Linnaeus, 1758) from Cyprus by 2D-PAGE. Arch. Toxicol. 2012, 86, (3), 441-451.

70. Serrano, S. M.; Shannon, J. D.; Wang, D.; Camargo, A.; Fox, J. W., A multifaceted analysis of viperid snake venoms by two-dimensional gel electrophoresis: An approach to understanding venom proteomics. Proteomics 2005, 5, (2), 501-510.

71. Ho, C.-L.; Ko, J.-L., Purification and characterization of a lethal protein with phospholipase A1 activity from the hornet (Vespa basalis) venom. Biochimica et Biophysica Acta (BBA) - Lipids and Lipid Metabolism 1988, 963, (3), 414-22.

72. Torres, A. F. C.; Huang, C.; Chong, C.-M.; Leung, S. W.; Prieto-da-Silva, A. R. B.; Havt, A.; Quinet, Y. P.; Martins, A. M. C.; Lee, S. M. Y.; Rádis-Baptista, G., Transcriptome analysis in venom gland of the predatory giant ant Dinoponera quadriceps: insights into the polypeptide toxin arsenal of Hymenopterans. PLoS One 2014, 9, (1), e87556.

73. dos Santos, L. D.; da Silva Menegasso, A. R.; dos Santos Pinto, J. R.; Santos, K. S.; Castro, F. M.; Kalil, J. E.; Palma, M. S., Proteomic characterization of the multiple forms of the PLAs from the venom of the social wasp Polybia paulista. Proteomics 2011, 11, (8), 1403-12.

74. Kini, R. M., Excitement ahead: structure, function and mechanism of snake venom phospholipase A 2 enzymes. Toxicon 2003, 42, (8), 827-840.

75. Xin, Y.; Choo, Y. M.; Hu, Z.; Lee, K. S.; Yoon, H. J.; Cui, Z.; Sohn, H. D.; Jin, B. R., Molecular cloning and characterization of a venom phospholipase $\mathrm{A} 2$ from the bumblebee Bombus ignitus. Comparative Biochemistry and Physiology Part B: Biochemistry and Molecular Biology 2009, 154, (2), 195-202.

76. Schmidt, J. O.; Blum, M. S.; Overal, W. L., Hemolytic activities of stinging insect venoms. Arch. Insect Biochem. Physiol. 1983, 1, (2), 155-60.

77. Schmidt, J. O.; Blum, M. S.; Overal, W. L., Comparative lethality of venoms from stinging Hymenoptera. Toxicon 1980, 18, (4), 469-74.

78. Safavi-Hemami, H.; Young, N. D.; Williamson, N. A.; Purcell, A. W., Proteomic interrogation of venom delivery in marine cone snails: novel insights into the role of the venom bulb. J. Proteome Res. 2010, 9, (11), 5610-19.

79. Bouzid, W.; Verdenaud, M.; Klopp, C.; Ducancel, F.; Noirot, C.; Vétillard, A., De Novo sequencing and transcriptome analysis for Tetramorium bicarinatum: a comprehensive venom gland transcriptome analysis from an ant species. BMC Genomics 2014, 15, (1), 987. 
80. Touchard, A.; Dejean, A.; Escoubas, P.; Orivel, J., Intraspecific variations in the venom peptidome of the ant Odontomachus haematodus (Formicidae: Ponerinae) from French Guiana. Journal of Hymenoptera Research 2015, 47, 87.

81. Prator, C. A.; Murayama, K. M.; Schulz, J. R., Venom variation during prey capture by the cone snail, Conus textile. PLoS One 2014, 9, (6), e98991.

82. Eskridge, E. M.; Elliott, W. B.; Elliott, A. H.; Eskridge, P. B.; Doerr, J. C.; Schneller, N.; Reisman, R. E., Adaptation of the electrical stimulation procedure for the collection of vespid venoms. Toxicon 1981, 19, (6), 893-97. 


\section{Figure Legends}

Figure 1. Representative MALDI-TOF MS spectra of $P$. clavata whole venom. Venom was obtained by electrical stimulation (Panel $A$ ) and manual dissection (Panels B and C) from colony 1 (panels A and B) and colony 2 (panel C). Initial screening was between 1000-10,000 $\mathrm{m} / \mathrm{z}$ (left-hand panels Aa-Ca). Right-hand panels show mass spectra between 1000-3500 $\mathrm{m} / \mathrm{z}$ where peptide abundance was the highest (panels $\mathrm{Ab}-\mathrm{Cb}$ ).

Figure 2. RP-HPLC chromatograms of $P$. clavata venom glands extracted by electrical stimulation or venom gland dissection. Venoms were separated by analytical C18 RP-HPLC using an ACN/0.085\% TFA gradient of 1\%/min (grey dotted line) at $1 \mathrm{ml} / \mathrm{min}$. Panels show the chromatographic elution profile of $P$. clavata venoms from colony 1 obtained by $(A)$ electrical stimulation and (B) manual dissection. Panel $C$ shows profile of venom from colony 2 obtained by manual dissection. Chromatographic peaks were monitored at 215 and 280 $\mathrm{nm}$ (only absorbance at $215 \mathrm{~nm}$ is shown for clarity). Peaks in which poneratoxin was found are labelled as "Potx".

Figure 3. LC-MALDI-TOF mass distribution and total peptide count of $P$. clavata venom obtained using the different venom collection methods. (A-C) Histograms show the abundance of peptide masses in each venom sorted into 100 Da mass bins (columns; lefthand ordinate axis) and the cumulative total of peptide masses in each venom (circles; righthand ordinate axis). Total peptide counts are indicated in the upper right-hand corner of each panel. (A) P. clavata venom obtained by electrical stimulation of ants from colony $1,(B)$ manual dissection of ants from colony 1 and (C) manually dissection of ants from colony 2 . Shaded areas represent $5-95 \%$ of the total number of peptides.

Figure 4 2D landscapes of $P$. clavata venom peptides from colony 1 determined using MALDI-TOF MS. Panels show venom peptides collected by (A) electrical stimulation, (B) manual dissection, and (C) peptides common to both collection methods based on a mass tolerance of $\pm 1 \mathrm{Da}$. Grey shaded areas represent $5-95 \%$ of peptide masses. Inset in panel C shows an area-proportional Euler plot depicting variation in numbers of shared and distinct peptides. Potx is represented by red circles. DV, manually dissected venom; ESV, electrically stimulated venom. (D) Mass list for peptides common to manually dissected and electrically stimulated venom. Potx is indicated by a red shaded box. 
Figure 5. 2D SDS-PAGE of reduced and alkylated $P$. clavata venoms. Venoms were extracted by (A) electrical stimulation of ants from colony 1 , (B) dissection of the venom glands of ants from colony 1 , and (C) dissection of the venom glands of ants from colony 2 . Representative gels from triplicates of each sample are shown. A total of (A) 53 spots, (B) 38 spots (circled) were excised for identification by nanoLC-ESI-QTOF MS/MS. In panel C 57 spots (circled) were observed, but no spots were unique from panels $A$ and B. Circles/ovals represent shared proteins between A, B and C (yellow), A and C (red) and B and C (green). Black circles/ovals represent unique proteins not detected in the other gels.

Figure 6. Functions of identified $P$. clavata venom proteins from colony 1 . A Pie graph depicting classification of venom proteins identified from 2D-PAGE spots using nanoLC-ESIQTOF MS/MS. Panels show venom proteins obtained by electrical stimulation (left-hand panel) and manually dissected venom (right-hand panel). B Area-proportional Euler plots depicting numbers of homologous and distinct venom proteins obtained by electrical stimulation (ESV; cyan circles) and manual dissection (DV; brown circles). 
For TOC Only

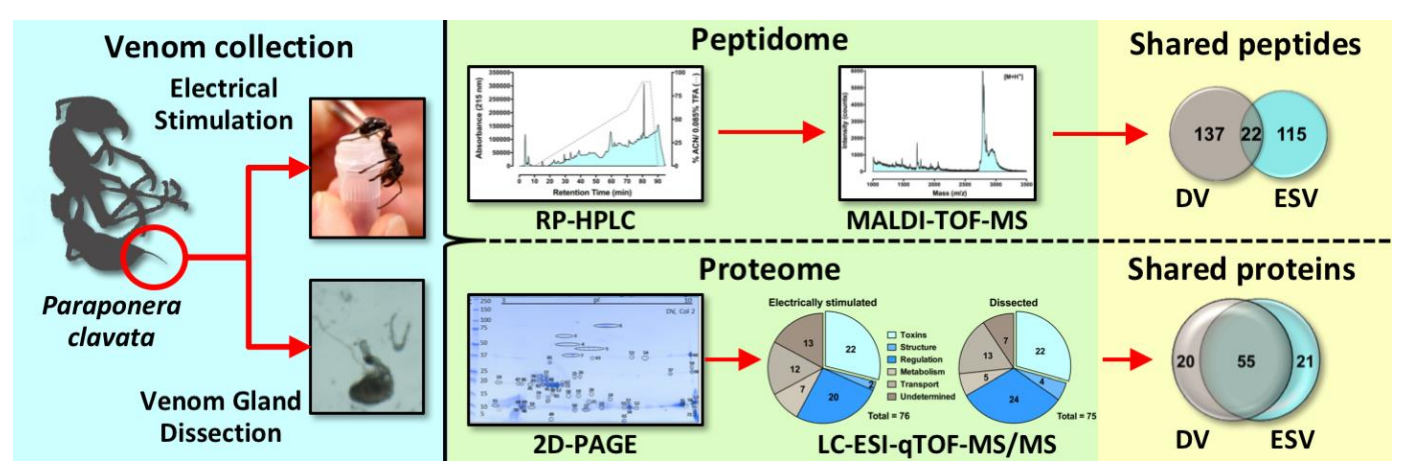

Photo courtesy of Axel Touchard 